\title{
Yield and enrichment studies of C-13 isotope by multi-photon dissociation of Freon-22 at low temperatures
}

\author{
MANOJ KUMAR $^{1} *$, ANANT DESHPANDE ${ }^{2}$, CHINTAN GUPTA $^{1}$ and \\ A K NATH ${ }^{1}$ \\ ${ }^{1}$ Centre for Advanced Technology, Indore 452 013, India \\ ${ }^{2}$ Devi Ahilya Vishwavidyalaya, Indore 452 001, India \\ e-mail: gmanoj@cat.ernet.in
}

MS received 11 March 2003; revised 17 May 2003

\begin{abstract}
Multi-photon dissociation of Freon-22 $\left(\mathrm{CF}_{2} \mathrm{HCl}\right)$ at low temperatures has been carried out to separate the $\mathrm{C}-13$ isotope using a TEA $\mathrm{CO}_{2}$ laser. Yield and enrichment of $\mathrm{C}-13$ isotope in the product $\mathrm{C}_{2} \mathrm{~F}_{4}$ are studied at $9 P(22)$ laser line as a function of temperature $\left(-50^{\circ} \mathrm{C}\right.$ to $\left.30^{\circ} \mathrm{C}\right)$. It is observed that at a given fluence when the temperature is lowered the yield decreases and the enrichment factor of $\mathrm{C}-13$ increases.

Room temperature irradiation of $\mathrm{CF}_{2} \mathrm{HCl}$ towards the blue edge of $\mathrm{C}-13$ absorption (i.e. at $9 P(20)$ laser line) gives low yield of the product $\left(\mathrm{C}_{2} \mathrm{~F}_{4}\right)$ at a fluence, which produces the desired enrichment factor of 100 . An increase in fluence gives very high yield of $\mathrm{C}_{2} \mathrm{~F}_{4}$ but the enrichment factor is very low. Irradiating $\mathrm{CF}_{2} \mathrm{HCl}$ at a temperature of $-10^{\circ} \mathrm{C}$ enhances the enrichment factor to 100 and the yield obtained is comparable to that towards the red edge of C-13 absorption (i.e. at $9 P(26)$ laser line).

At a given enrichment factor higher enrichment efficiency is achieved when $\mathrm{CF}_{2} \mathrm{HCl}$ is irradiated at lower temperature.
\end{abstract}

Keywords. TEA $\mathrm{CO}_{2}$ laser; carbon-13; isotope separation; low temperature.

\section{Introduction}

Soon after the discovery of TEA $\mathrm{CO}_{2}$ lasers, it was demonstrated ${ }^{1}$ that highly monochromatic and intense infrared radiation could interact with polyatomic molecules possessing strong IR absorption bands, leading to absorption of many photons resulting in its dissociation. This process is termed as infra-red multi photon dissociation (IRMPD). The process of multi-photon decomposition, which could be isotope selective, triggered a great interest in separating a variety of isotopes by laser method known as laser isotope separation (LIS). In this method the molecules bearing desired isotopes are dissociated by monochromatic and intense laser radiation, leaving the other isotopebearing molecules almost untouched. LIS of C-13 isotopes by IRMPD of Freon-22 $\left(\mathrm{CF}_{2} \mathrm{HCl}\right)$ is a very promising method ${ }^{2,3}$ in which the $9 P$ band laser lines of pulsed $\mathrm{CO}_{2}$ laser can be selectively absorbed by $\mathrm{C}-13$ bearing $\mathrm{CF}_{2} \mathrm{HCl}$ molecules leading to this dissociation and formation of the product $\left(\mathrm{C}_{2} \mathrm{~F}_{4}\right)$, enriched in $\mathrm{C}-13$ isotopes. The process of selective dissociation of $\mathrm{CF}_{2} \mathrm{HCl}$ is described as,

*For correspondence 


$$
\begin{aligned}
& { }^{13} \mathrm{CF}_{2} \mathrm{HCl}+n h v \rightarrow \frac{1}{2}{ }^{13} \mathrm{C}_{2} \mathrm{~F}_{4}+\mathrm{HCl}, \\
& { }^{12} \mathrm{CF}_{2} \mathrm{HCl} \rightarrow \text { almost no reaction. }
\end{aligned}
$$

Depending upon the $\mathrm{CO}_{2}$ laser line in the $9 P$ band and its fluence, the most abundant isotope of carbon (C-12) absorbs much less and may dissociate with about 100 times smaller probability giving the product $\mathrm{C}_{2} \mathrm{~F}_{4}$ an isotopic ratio of $1: 1$ or an enrichment factor $\beta$ of 100 . We are also aiming here at a $\beta$ of 100 .

In our previous work ${ }^{4}$ related to maximization of yield of C-13 isotope, we have demonstrated that the enrichment efficiency at room temperature is maximum when Freon samples are irradiated towards the red edge of the absorption curve of $\mathrm{C}-13$ bearing $\mathrm{CF}_{2} \mathrm{HCl}$ molecules. To reduce the contribution of $\mathrm{C}-12$ we shifted towards the red edge and irradiated Freon samples at higher fluences giving higher yields of $\mathrm{C}_{2} \mathrm{~F}_{4}$ at a $\beta$ value of 100. These results encouraged us to go for low temperature irradiation of Freon at laser lines $(9 P(18)$ or $9 P(20))$, which are better absorbed in Freon. At these laser lines and at room temperature, a larger contribution of $\mathrm{C}-12$ restricts the fluence to a lower value at $\beta$ of 100 , giving lower yields of $\mathrm{C}_{2} \mathrm{~F}_{4}$. At the same time the enrichment efficiency is poor. In order to reduce the contribution of undesired isotope to the yield of dissociation products, low temperature irradiation was carried out. The aim was to exploit better absorption of these lines, thus increasing the yield of the product whereas lowering the temperature could control selectivity and hence the enrichment factor. In this paper we report low temperature irradiation of Freon towards the blue edge of the $\mathrm{C}-13$ isotope, aimed towards higher yields of $\mathrm{C}_{2} \mathrm{~F}_{4}$ at a $\beta$ value of 100. At low temperature, higher enrichment efficiency is also expected towards the blue edge because of higher operating fluence at given $\beta$.

\section{IRMPD at low temperature}

The natural abundance $(\alpha)$ of $\mathrm{C}-13$ is only $1 \cdot 11 \%$. The isotopic shift between ${ }^{12} \mathrm{CF}_{2} \mathrm{HCl}$ and ${ }^{13} \mathrm{CF}_{2} \mathrm{HCl}$ molecules is $24 \mathrm{~cm}^{-1}$. The $\mathrm{C}-13$ bearing ${ }^{13} \mathrm{CF}_{2} \mathrm{HCl}$ molecules can be selectively dissociated by an appropriate choice of laser line in the $9 P$ band of a $\mathrm{CO}_{2}$ laser.

Depending upon the ratio of C-13 and C-12 bearing molecules in the product $\left(\mathrm{C}_{2} \mathrm{~F}_{4}\right)$ and in the reactant $\left(\mathrm{CF}_{2} \mathrm{HCl}\right)$ the enrichment factor ${ }^{5} \beta$ is defined as:

$$
\beta=\left({ }^{13} \mathrm{C} /{ }^{12} \mathrm{C}\right) \text { product } /\left({ }^{13} \mathrm{C} /{ }^{12} \mathrm{C}\right) \text { reactant. }
$$

Since we have used QMA to detect the product at mass numbers 81,82 and 83 corresponding to ${ }^{12} \mathrm{C}-{ }^{12} \mathrm{C},{ }^{12} \mathrm{C}-{ }^{13} \mathrm{C}$ and ${ }^{13} \mathrm{C}-{ }^{13} \mathrm{C}$ atom combinations respectively, the formula for enrichment factor ${ }^{6,7}$ becomes,

$$
\beta=\{(1-\alpha) / \alpha\}\left\{\left[2 P_{83}+P_{82}\right] /\left[2 P_{81}+P_{82}\right]\right\},
$$

where $P_{81}, P_{82}$ and $P_{83}$ are partial pressures corresponding to mass numbers 81,82 and 83 respectively of $\mathrm{C}_{2} \mathrm{~F}_{3}{ }^{+}$ions as detected by quadrapole mass analyzer (QMA).

To compare various results we measured the total quantity of $\mathrm{C}_{2} \mathrm{~F}_{4}$ produced after $m$ number of pulses using QMA and termed it overall yield $X{ }^{4}$ A higher overall yield of $\mathrm{C}_{2} \mathrm{~F}_{4}$ at desired $\beta$ automatically ensures higher yield of the $\mathrm{C}-13$ isotope. The yield of the $\mathrm{C}-13$ isotope however refers to the ratio of $\mathrm{C}-13$ bearing molecules dissociated to the number of them irradiated per pulse. 
At room temperature the fluence at a given laser line has a maximum value in order to limit the yield of undesired isotope so that the desired enrichment factor could be achieved in the product. Since the yield of the product $\left(\mathrm{C}_{2} \mathrm{~F}_{4}\right)$ is a nonlinear function of the fluence it is desired to increase the operating fluence to get its higher yield. However, to maintain desired enrichment factor in the product, which tends to drop sharply with increase in fluence, there are two ways:

(a) Irradiation at wavelength towards the red edge of absorption of C-13 isotope. This option has been already discussed in detail in our previous work. ${ }^{4}$

(b) Irradiation at low temperature. To highlight the advantages of low temperature LIS technique we would like to refer to sulphur isotope separation at low temperatures. ${ }^{8}$ The salient features of low temperature MPD are as follows.

(i) Narrowing down of the multi-photon absorption curve.

The width of absorption curve is determined by the population distribution of molecules in different rotational levels of ground electronic vibrational state. Lowering of temperature reduces the population (proportional to $\exp (-\Delta E / k T)$, where $\Delta E$ is the energy of the level, $k$ is the Boltzmann constant and $T$ is temperature of molecules) of higher rotational levels more than low lying rotational levels. The population of higher rotational levels contributes to red edge of the absorption curve so reduction in temperature narrows the absorption curve preferentially in its red edge.

(ii) Decrease of multi-photon absorption coefficient at a given line.

(iii) Reduction in the red edge of the absorption curve to a great extent compared to the blue edge with the lowering of the temperature.

These points will be referred to again in $\$ 4$ to explain the results.

\section{Experimental setup}

The multi-photon dissociation of $\mathrm{CF}_{2} \mathrm{HCl}$ was carried out using line tunable high repetition rate $\mathrm{UV}$ pre-ionized TEA $\mathrm{CO}_{2}$ laser described earlier. ${ }^{9}$ The resonator consists of a curved $\mathrm{ZnSe}$ output coupler (reflectivity $=85 \%$ ) of $5 \mathrm{~m}$ radius of curvature (ROC) and a grating having 150 lines per mm. Various lines in the 9.6 and 10.4 micron bands can be tuned by changing the angle of the grating. The resonator is isolated from the laser discharge by a pair of Brewster windows. The laser can be operated at $1-500 \mathrm{~Hz}$ repetition rate. The pulse-to-pulse stability is $\pm 1.4 \%$.

The laser emits pulses with an initial spike of about $130 \mathrm{~ns}$ (FWHM) duration followed by a long tail. The energy content in the tail is governed by the amount of $\mathrm{N}_{2}$ in the laser mixture. A small amount of nitrogen is added to the laser mixture to improve its efficiency and at the same time energy appearing in the tail is insignificant. A little amount of $\mathrm{H}_{2}$ is also added to the laser mixture to improve the stability of discharge and pulse energy. The partial pressures of various gases i.e. $\mathrm{CO}_{2}, \mathrm{~N}_{2}, \mathrm{He}$ and $\mathrm{H}_{2}$ are 140, 10, 630 and 15 mbar respectively. Since the raw beam fluence is much smaller than that required for carrying out multi-photon decomposition, the beam is focused with a mirror of $50 \mathrm{~cm}$ radius of curvature, kept at an angle. The interaction takes place near focal zone in a small irradiation cell of volume $240 \mathrm{cc}$. The schematic diagram of $\mathrm{TEA} \mathrm{CO}_{2}$ laser and the irradiation cell having the provision to maintain low temperature is shown in figure 1. 


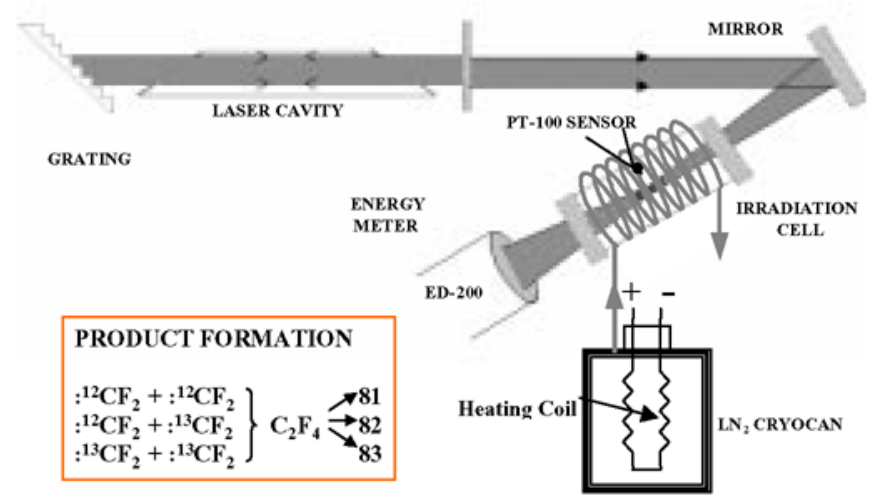

Figure 1. Schematic drawing of the C-13 isotope separation process at low temperature.

To maintain low temperature in the irradiation cell a hollow copper tube is wrapped over the cell and liquid nitrogen vapours are allowed to pass through it. Liquid nitrogen vapour is continuously produced by boiling liquid nitrogen with the help of a heater placed in the liquid nitrogen cryogenic can.

Any desired temperature as low as $-80^{\circ} \mathrm{C}$ can be maintained with an accuracy of $\pm 1^{\circ}$. A PT-100 sensor measures the temperature of the gas in the cell.

\section{Results}

To study the general behaviour of $X$ and $\beta$ at low temperatures, irradiation of Freon-22 samples were carried out at $9 P(22)$ laser line from room temperature down to $-50^{\circ} \mathrm{C}$. Freon samples were irradiated at constant fluence for $1.5 \mathrm{~h}$ at a repetition rate of $6 \mathrm{~Hz}$. The results of the experiments at low temperature are presented in figure 2. At a constant fluence when the temperature is lowered the enrichment factor of $\mathrm{C}-13$ increases while the yield of $\mathrm{C}_{2} \mathrm{~F}_{4}$ decreases. The reason for increase in selectivity and hence $\beta$ is due to narrowing down of the multi-photon absorption curve at low temperature as mentioned in the previous section. When $\mathrm{C}-13$ is preferentially excited the selectivity of the process depends upon the participation of $\mathrm{C}-12$ bearing molecules, which is due to the tail portion, i.e. the red edge of the $\mathrm{C}-12$ absorption curve. Since the red edge of the absorption curve is mainly due to hot band contribution, it reduces significantly as the Freon-22 sample temperature is reduced. There is also reduction in the yield of C-13 but the reduction is greater for $\mathrm{C}-12$, thus the selectivity, which is the ratio of the yield of $\mathrm{C}-13$ to that of $\mathrm{C}-12$, increases with reduction in temperature resulting in increase in enrichment factor of $\mathrm{C}-13$ isotope in the product. The reduction in yield of $\mathrm{C}_{2} \mathrm{~F}_{4}$ is due to reduction in absorption coefficient at low temperature compared to that at room temperature.

The enrichment efficiency, which basically gives an idea of laser energy spent per C-13 atom separated, can also be logically inferred at a given $\beta$ for two enrichment conditions (irradiation at room temperature at lower fluence and at low temperature at higher fluence) in a similar fashion as discussed earlier. ${ }^{4}$ 
In order to increase the enrichment efficiency at a $\beta$ value of 100 towards the blue edge of $\mathrm{C}-13$ absorption (i.e. $9 P(20)$ laser line), the operating fluence was increased and Freon22 sample temperature was reduced to control $\beta$. The results are tabulated in table 1.

It is observed that at room temperature and at higher fluence $(\mathbf{C})$, the yield of $\mathrm{C}_{2} \mathrm{~F}_{4}$ is very large but the $\beta$ is very poor. To increase $\beta$, we therefore reduced the temperature such that the $\beta$ value of 100 could be achieved. At $-10^{\circ} \mathrm{C}$ the enrichment factor of 100 is obtained (B) but the yield of $\mathrm{C}_{2} \mathrm{~F}_{4}$ has also reduced compared to its room temperature value. The enrichment efficiency however has increased as the enhancement in yield is much more compared to increase in the optimum fluence at $\beta$ value of 100 (compare the fluence of (B) and (D) and their respective yields). The increase in enrichment efficiency is again due to nonlinear ${ }^{10}$ dependence of the yield on fluence. Low temperature allows high operating fluence at desired $\beta$ thus increasing the enrichment efficiency. Moreover at low temperature and towards blue edge (at $9 P(20)$ line), yield comparable to that as obtained towards red edge (at $9 P(26)$ line) at room temperature has been obtained (compare results $(\mathbf{A})$ and $(\mathbf{B})$ ).

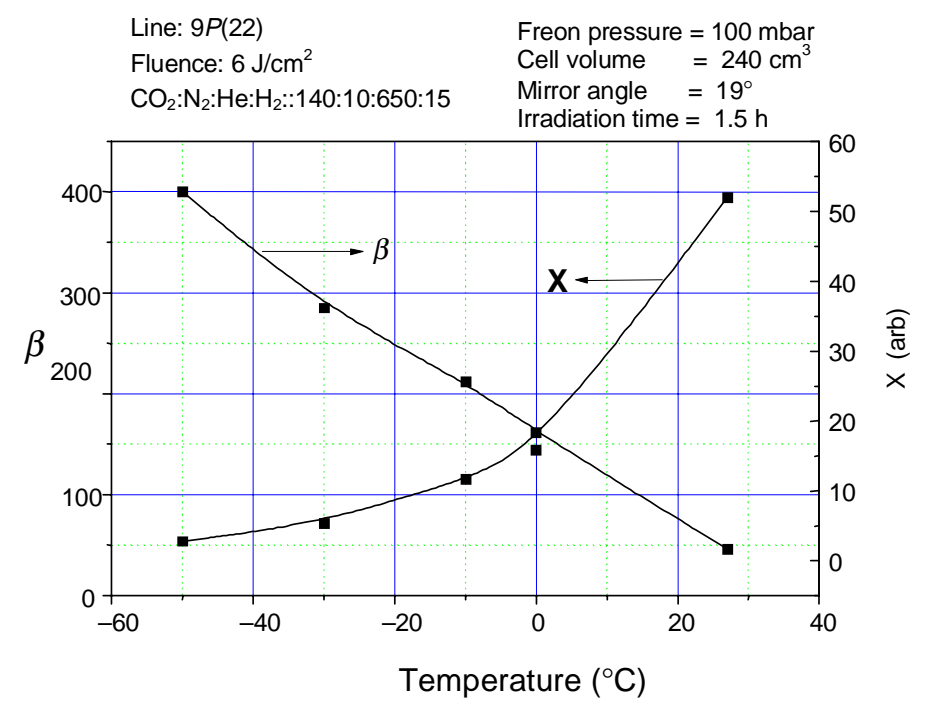

Figure 2. Variation of $\beta$ and $X$ at $9 P(22)$ laser line as a function of temperature.

Table 1. Comparison of overall yield of $\mathrm{C}_{2} \mathrm{~F}_{4}$ and enrichment factor for $9 P(20)$ and $9 P(26)$ laser lines at different temperatures.

Freon pressure $=100 \mathrm{mbar}$, irradiation time $=1.0 \mathrm{~h}$.

\begin{tabular}{lcccrc}
\hline Results & $\begin{array}{c}\text { Laser line } \\
(\mu \mathrm{m})\end{array}$ & $\begin{array}{c}\text { Fluence } \\
\left(\mathrm{J} / \mathrm{cm}^{2}\right)\end{array}$ & $\begin{array}{c}X \\
(\text { a.u. })\end{array}$ & $\beta$ & $\begin{array}{c}\text { Temperature } \\
\left({ }^{\circ} \mathrm{C}\right)\end{array}$ \\
\hline A & $9 P(26)(1041 \cdot 279)$ & $7 \cdot 65$ & 17.9 & 100 & 30 \\
B & $9 P(20)(1046 \cdot 874)$ & $5 \cdot 37$ & 12.6 & 100 & -10 \\
C & $9 P(20)(1046 \cdot 874)$ & $5 \cdot 37$ & 24.5 & 24 & 30 \\
D & $9 P(20)(1046 \cdot 874)$ & 3.40 & $6 \cdot 66$ & 100 & 30 \\
\hline
\end{tabular}




\section{Conclusions}

From the above studies the following conclusions can be drawn.

(1) Lowering of temperature increases the selectivity and lowers the yield of isotope at a given fluence.

(2) Maximum fluence for $\beta$ equal to 100 is low when LIS towards blue edge of C-13 is carried out at room temperature. This results in low enrichment efficiency. The maximum fluence increases with lowering of the temperature. This increases the enrichment efficiency.

(3) The key to higher enrichment efficiency lies in higher operating fluence which is possible by irradiating the Freon samples towards the red edge of C-13 absorption ${ }^{4}$ or by irradiating at low temperature. In both cases at higher fluence the participation of C-12 to the yield of $\mathrm{C}_{2} \mathrm{~F}_{4}$ is reduced.

\section{Acknowledgements}

Authors wish to acknowledge with gratitude the constant guidance, encouragement and support of Dr D D Bhawalkar in this work. They would also like to thank Drs N Venkatramani, S K Sarkar and V Parthasarathy, Bhabha Atomic Research Centre, Mumbai for their invaluable suggestions in setting up the C-13 of isotope experiments and diagnostics. The technical assistance of Mr Prabhu Sangale in carrying out the experiments is also acknowledged.

\section{References}

1. Isenor N R and Richardson M C 1971 Appl. Phys. Lett. 18224

2. Gautheir M, Cureton C G, Hackett P A and Willis C 1982 Appl. Phys. B28 43

3. Outhouse A, Lawrence P, Gauthier M and Hackett P A 1985 Appl. Phys. B36 63

4. Kumar M, Deshpande A, Gupta C, Biswas A K and Nath A K 2002 Proc. Indian Acad. Sci. (Chem. Sci.) 114659

5. Parthasarathy V, Nad S, Annaji Rao K and Sarkar S K 1997 J. Photochem. Photophys. A110 11

6. Parthasarathy V, Sarkar S K, Iyer N V, Rama Rao K V S and Mittal J P 1993 Appl. Phys. B56 321

7. Ivanenko M, Handreck H, Gothel J, Fuss W, Kompa K L, Schmid W E and Witte K 1997 Appl. Phys. B65 577

8. Baranov Y Yu, Velikhov E P, Kolomiiskii Yu R, Letokhov V S, Nizev V N, Pismennyi V D and Ryabov E A 1995 Sov. J. Quantum Electron. 9621

9. Kumar M, Choudhary P, Shailesh Tiwari and Nath A K 1998 Proc. Int. Conf. on Optics and Optoelectronics (ICOL-98) (New Delhi: Narosa) pp 1017-1020

10. Gothel J, Ivanenko M, Kompa K L and Schmid W E 1994 Atoms, Molecules Clusters 2447 